DOI: $10.12731 / 2306-1561-2013-4-29$

\title{
MODELING THE DEVELOPMENT OF ENTERPRISES IN THE CONDITIONS OF COMPETITION
}

Kotov A.P., Bogdanov B.M.

\begin{abstract}
The current economic situation in the country is characterized by the struggle of the enterprises for survival. Any enterprise consumes resources, manufactures products and is expanding at the expense of profits. If the enterprise in parallel, there are a variety of production, in the conditions of market economy, between them there is competition for resources (equipment, materials, staff, etc.). As a result, production that is more efficient is developing faster and suppresses competitor. This process is shaped rather reminds the development of biological communities in which there is competition between different modes. This article makes an attempt to simulate such economic processes with the help of wellknown mathematical models in biology and ecology.
\end{abstract}

Keywords: modeling, mathematical model, enterprise, competition, biological communities.

\subsection{2}

\section{МОДЕЛИРОВАНИЕ РАЗВИТИЯ ПРЕДПРИЯТИЙ В УСЛОВИЯХ КОНКУРЕНЦИИ}

\section{Котов А.П., Богданов Б.М.}

\section{Аннотация}

Для современной экономической ситуаџии в стране характерна борьба предприятий за выживание. Любое предприятие потребляет ресурсы, производит продукиию и расширяется за счет получаемой прибыли. Если на предприятии параллельно существуют различные производства, то в условиях рыночной экономики между ними возникает конкуренция за ресурсы (площади, оборудование, материаль, персонал и т.п.). В результате более эффективное производство развивается быстрее u подавляет конкурента. Этот прочесс по форме очень напоминает развитие биологических сообществ, в которых имеется межвидовая конкуренция. В статье предпринята попытка моделирования подобных экономических процессов с помощью известных математических моделей из области биологии и экологии.

Ключевые слова: моделирование, математическая модель, предприятие, конкуренция, биологические сообщества. 


\section{Введение}

Для современной экономической ситуации в стране характерна борьба предприятий за выживание. Любое предприятие потребляет ресурсы, производит продукцию и расширяется за счет получаемой прибыли. Если на предприятии параллельно существуют различные производства, то в условиях рыночной экономики между ними возникает конкуренция за ресурсы (площади, оборудование, материалы, персонал и т.п.). В результате более эффективное производство развивается быстрее и подавляет конкурента [1, 4 - 6]. Этот процесс по форме очень напоминает развитие биологических сообществ, в которых имеется межвидовая конкуренция. Поэтому у авторов и появилась идея использовать для моделирования подобных экономических процессов некоторые известные математические модели из области биологии и экологии [1].

\section{Простейшая модель развития}

Развитие биологической популяции без конкуренции и при неограниченных ресурсах описывается следующим уравнением [1]

$$
\frac{d N}{d t}=r \cdot N
$$

где N - количество особей,

$\mathrm{t}$ - время,

$\mathrm{r}$ - мгновенная удельная скорость роста популяции, или коэффициент размножения, показывающий сколько себе подобных производит одна особь в единицу времени.

Применим уравнение (1) для описания развития предприятия в условиях отсутствия конкуренции и при неограниченных ресурсах. Под $\mathrm{N}$ будем понимать производственную мощность или объём производимой продукции за отчётный период, a под $\mathrm{r}$ - скорость расширения производства или относительное увеличение производственной мощности в единицу времени, достигаемое за счёт прибыли, полученной от реализации единицы продукции

$$
r=\Pi_{0} \cdot \frac{k_{p}}{3_{0}},
$$

где П - прибыль от реализации единицы продукции (удельная прибыль),

$\mathrm{k}_{\mathrm{p}}$ - относительная доля прибыли отчисляемая на расширение производства,

$3_{0}$ - затраты необходимые для увеличения производственной мощности на единицу продукции (удельные затраты на расширение производства).

Объём производимой продукции можно выражать или в стоимостном или в натуральном виде. В первом случае размерность $\mathrm{N}$ будет руб/год, $\Pi_{0}$ - руб/руб, $3_{0}$ руб/руб/год. Во втором случае имеем $\mathrm{N}-$ шт/год, $3_{0}$ - руб/шт/год. При это м в обоих случаях коэффициент $\mathrm{r}$ имеет одну и туже размерность, обратную времени - 1/год, что соответствует уравнению (1). Поскольку r, как видно из формулы (2) характеризует 
эффективность производства данного вида продукции, будем в дальнейшем называть его также коэффициентом эффективности.

Отметим одну особенность, связанную с применением уравнения (1). При его выводе (применительно к биологическим объектам) предполагалось, что каждая особь с момента своего рождения непрерывно производит себе подобных со скоростью $\mathrm{r}$. Хотя это и является определённым упрощением реального процесса, адекватность математической модели существенно не нарушается, особенно для простейших организмов. Однако при развитии предприятия процесс носит трехступенчатый характер: сначала на имеющейся производственной мощности $(\mathrm{N})$ за определённый промежуток времени (dt) производится товарная продукция (объёмом $\mathrm{N} \bullet \mathrm{dt}$ ), затем она реализуется и приносит прибыль $\left(\Pi_{0} \bullet \mathrm{N} \bullet \mathrm{dt}\right)$, часть которой $\left(\Pi_{0} \bullet \mathrm{kp} \bullet \mathrm{N} \bullet \mathrm{dt}\right)$ вкладывается в расширение производства и в результате этого увеличивается производственная мощность

$$
d N=\left(\Pi_{0} \frac{k_{p}}{3_{0}}\right) N d t=r N d t .
$$

По внешнему виду уравнение (3) аналогично уравнению (1). Но, по сути, эти уравнения будут эквивалентны только при условии, что все три перечисленных этапа процесса развития предприятия не имеют разрыва во времени. В отношении производства и реализации продукции это, как правило, соответствует действительности. Расширение производства в современных условиях также стараются проводить максимально быстро, за счет приобретения большого количества материалов и комплектующих, дополнительного набора персонала и задействования резервного оборудования, без капитального строительства, влекущего значительные затраты времени. Поэтому уравнение (1), на наш взгляд, вполне применимо для исследования динамики развития предприятия. Во всяком случае, погрешности будут не больше, чем при анализе развития популяций, для которого это уравнение широко используется.

Решением уравнений как (1), так и (3) является экспоненциальная функция

$$
N=N_{0} \cdot e^{r t}
$$

где $\mathrm{N}_{0}$ - начальное значение величины $\mathrm{N}$ (при $\mathrm{t}=0$ ).

Очевидно, что рост $\mathrm{N}$ не ограничен. Это вполне естественно, так как решение (4) описывает процесс самовоспроизводства при отсутствии каких-либо противодействий или ограничений.

Данная экономическая трактовка уравнения (1) справедлива, прежде всего, для рыночных отношений. В этом случае движущей силой процесса является получение прибыли, расширенное воспроизводство и получение ещё большей прибыли. Коэффициент r и, в частности, входящая в него удельная прибыль П 0 определяются объективными факторами, например нормальной рыночной конкуренцией между предприятиями или специальными антимонопольными мерами со стороны государства. Однако, как пока что показывает наш отечественный опыт, предприятия зачастую увеличивают объём производимой продукции только в денежном выражении - за счет повышения цен и без какого-либо расширения производства. В таких условиях 
рассмотренная модель, разумеется, неприемлема. Однако такие условия не являются нормальными и рано или поздно будут изменены.

\section{Модель развития при ограниченных ресурсах}

Развитие биологической популяции при ограниченных ресурсах описывается так называемым логистическим уравнением

$$
\frac{d N}{d t}=\frac{r N(K-N)}{K}
$$

где $\mathrm{K}$ - предельно возможная величина популяции $(\mathrm{N}-\mathrm{K})$.

Сомножитель (K-N)/K, которым это уравнение отличается от уравнения (1), учитывает переменный характер скорости размножения - чем ближе величина популяции $\mathrm{N}$ к пределу $\mathrm{K}$, тем меньше скорость размножения. В пределе при $\mathrm{N}=\mathrm{K}$ она превращается в ноль.

Дадим несколько иную трактовку уравнению (5), которая будет полезна для дальнейшей экономической интерпретации. Пусть для существования рассматриваемого вида на одну особь необходим некоторый удельный ресурс, а в наличии имеется суммарный объём данного ресурса $\mathrm{S}$. Тогда очевидно

$$
K=\frac{S}{s}, \frac{K-N}{K}-\frac{\frac{S}{s}-N}{\frac{S}{s}}=\frac{S-N \cdot s}{S}=\frac{\Delta S}{S},
$$

где $\Delta \mathrm{S}$ - неиспользованный остаток необходимого ресурса.

Таким образом, множитель (К-N)/К можно рассматривать как относительную долю неизрасходованного ресурса. Другими словами, скорость воспроизводства прямо пропорциональна остающейся в наличии свободной части ресурса. Это вполне понятно, так как чем меньше остаётся ресурсов, тем медленнее должно происходить размножение.

Сказанное можно перенести и на развитие предприятия. Любой необходимый для этого ресурс теоретически имеет верхнюю границу. Чем ближе мы к ней подходим, тем больших затрат это требует. Например, если речь идёт о расширении производственных площадей, то сначала этого можно достичь просто за счёт использования пустующих площадей. Затем - за счет перепланировки и перестановки оборудования, затем - за счёт реконструкции производственных помещений и т.п. Каждый последующий шаг требует увеличения удельных затрат на расширение производства $\left(3_{0}\right)$. То есть фактически коэффициент $r(2)$ будет убывать с ростом $\mathrm{N}$, что качественно соответствует уравнению (5).

Итак, пусть S - некоторый максимально возможный ресурс, например общая производственная площадь, a s - удельная потребность в этом ресурсе (в нашем случае это производственная площадь необходимая для выпуска единицы продукции за отчетный период). Тогда уравнение (5) можно переписать в виде

$$
\frac{d N}{d t}=\frac{r \cdot N(S-N s)}{S} .
$$


При этом $\mathrm{K}=\mathrm{S} / \mathrm{s}$ будет означать предельно достижимую в данных условиях производственную мощность.

Если для производства продукции требуется несколько разнородных ресурсов, характеризующихся параметрами si и $\mathrm{Si}$, то

$$
K=\min \frac{S_{i}}{s_{i}}
$$

а в уравнение (6) вместо s и $\mathrm{S}$ надо подставлять значения, соответствующие наиболее критичному ресурсу, исходя из которого определяется параметр К. В дальнейшем такой ресурс будем называть лимитирующим.

\section{Модель развития с учётом конкуренции}

Теперь рассмотрим развитие сообщества, состоящего из двух конкурирующих между собой видов. Процесс описывается математической моделью Лотки-Вольтерры [1]:

$$
\left\{\begin{array}{l}
\frac{d N_{1}}{d t}=\frac{r_{1} \cdot N_{1}\left(K_{1}-\left(N_{1}+\alpha_{12} N_{2}\right)\right)}{K_{1}} \\
\frac{d N_{2}}{d t}=\frac{r_{2} N_{2}\left(K_{2}-\left(N_{2}+\alpha_{21} N_{1}\right)\right)}{K_{2}} .
\end{array}\right.
$$

Физический смысл этих уравнений такой же, как и уравнения (5). Индексами 1 и 2 обозначены параметры, относящиеся к первому и второму виду соответственно. Если параметры $K_{1}$ и $K_{2}$ характеризуют ограничения по ресурсам, то коэффициенты $\alpha_{12}$ и $\alpha_{21}$ - межвидовую борьбу, т.е. показывают в какой степени один вид подавляется другим.

Система уравнений (8) решается численными методами. Однако ряд качественных результатов можно установить более простым путем. Следуя работе [1], рассмотрим плоскость с осями координат, по которым будем откладывать величины $\mathrm{N}_{1}$ и $\mathrm{N}_{2}$. Каждая точка плоскости с координатами $\mathrm{N}_{1}$ и $\mathrm{N}_{2}$ характеризует некоторое состояние сообщества. Линии в этой плоскости являются траекториями его развития. Начинается такая линия в точке с координатами $\mathrm{N}_{10}$ и $\mathrm{N}_{20}$ (значения $\mathrm{N}_{1}$ и $\mathrm{N}_{2}$ при $\mathrm{t}=0$ ), a заканчивается в точке, соответствующей устойчивому состоянию сообщества $\mathrm{N}_{1 \kappa}, \mathrm{N}_{2 \kappa}$ (если оно в принципе достижимо). Направление траектории развития, как следует из уравнения (8), для каждой точки плоскости определяется однозначно, так что различные траектории развития между собой пересекаться не могут.

Рассмотрим условия существования устойчивого состояния в сообществе из двух конкурирующих видов. Необходимое условие - это очевидно:

$$
\frac{d N_{1}}{d t}=\frac{d N_{2}}{d t}=0
$$

Как видно из уравнений (8) 


$$
\left\{\begin{array}{l}
\frac{d N_{1}}{d t}=0 \text { при } \mathrm{K}_{1}-N_{1}-\alpha_{12} N_{2}=0, \\
\frac{d N_{2}}{d t}=0 \text { при } \mathrm{K}_{2}-N_{2}-\alpha_{21} N_{2}=0 .
\end{array}\right.
$$

Эти прямые будем называть характеристическими. Кроме того, очевидно, что соотношение $\mathrm{dN}_{1} / \mathrm{dt}=0$ выполняется на оси ординат, $\mathrm{a} \mathrm{dN}_{2} / \mathrm{dt}=0-$ на оси абсцисс.

В зависимости от значений параметров $\mathrm{K}_{1}, \mathrm{~K}_{2}, \alpha_{12}, \alpha_{21}$ возможны четыре варианта взаимного расположения характеристических прямых на плоскости (рисунок $1)$ :

вариант «а»- $\mathrm{K}_{1}>\mathrm{K}_{2} / \alpha_{21}$ и $\mathrm{K}_{2}<\mathrm{K}_{1} / \alpha_{12}$,

вариант «б»- $\mathrm{K}_{1}<\mathrm{K}_{2} / \alpha_{21}$ и $\mathrm{K}_{2}>\mathrm{K}_{1} / \alpha_{12}$,

вариант «в»- $\mathrm{K}_{1}>\mathrm{K}_{2} / \alpha_{21}$ и $\mathrm{K}_{2}>\mathrm{K}_{1} / \alpha_{12}$,

вариант «г»- $\mathrm{K}_{1}<\mathrm{K}_{2} / \alpha_{21}$ и $\mathrm{K}_{2}<\mathrm{K}_{1} / \alpha_{12}$.
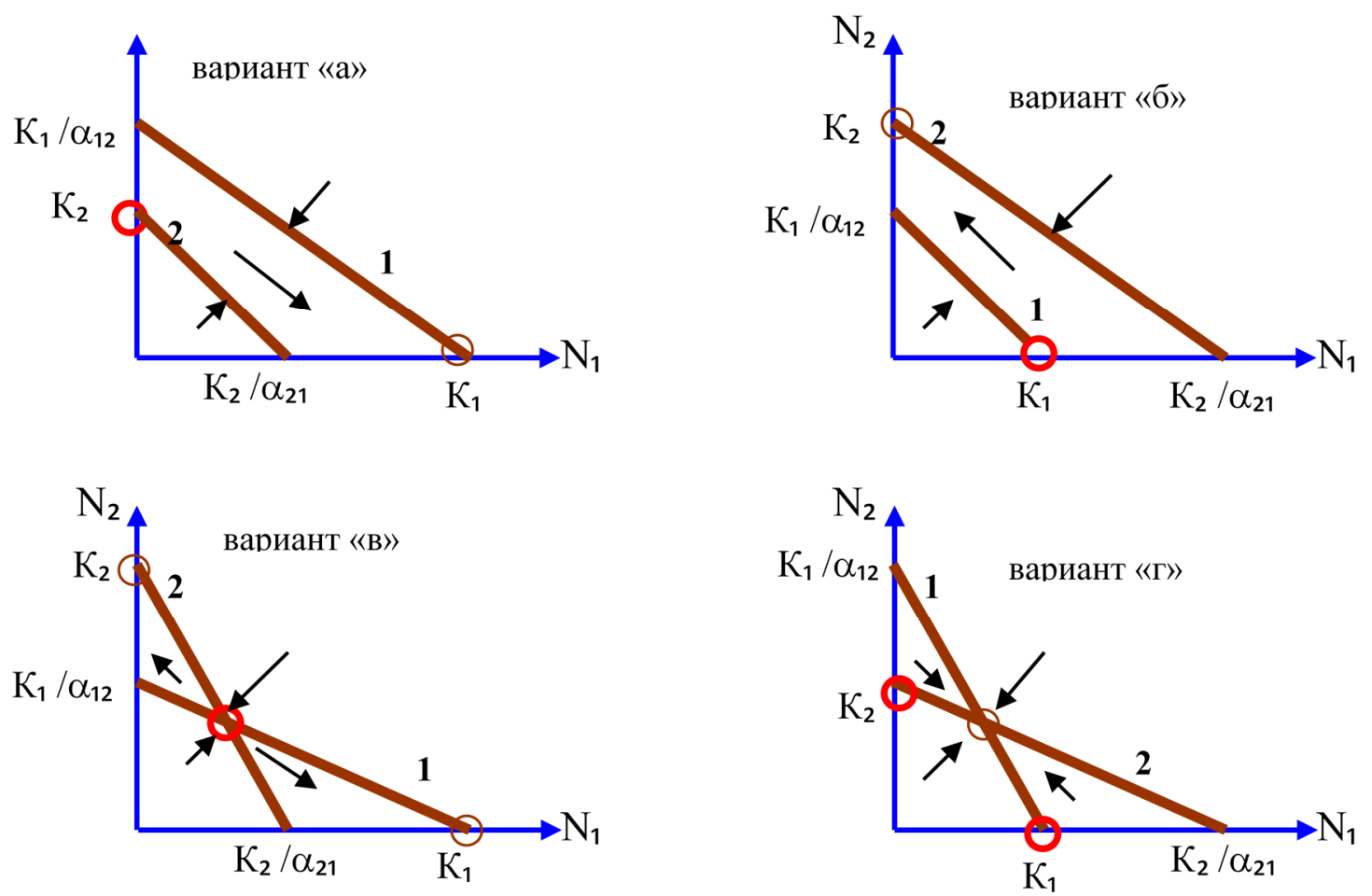

Рисунок 1 - Различные варианты развития сообществ, состоящих из двух конкурирующих видов:

$$
1-\mathrm{dN}_{1} / \mathrm{dt}=\mathbf{0}
$$

$$
2-\mathrm{dN}_{2} / \mathrm{dt}=\mathbf{0}
$$

\section{- устойчивое состояние,}

\section{- неустойчивое состояние.}

Кружками на рисунке1 обозначены точки, в которых выполняется условие (9). 
Направление развития процесса в каждой точке плоскости однозначно характеризуется вектором $\overrightarrow{d N}$, компонентами которого являются векторы $\overrightarrow{d N}_{1}$ и $\overrightarrow{d N}_{2}$ соответственно. Знаки $\overrightarrow{d N}_{1}$ и $\overrightarrow{d N}_{2}$ практически без вычислений определяются из уравнений (8), а с их учётом - преобладающие направления развития процесса для всех характерных областей в плоскости $\mathrm{N}_{1} \mathrm{~N}_{2}$ (на рис. 1 показаны стрелками). Это позволяет определить, является ли устойчивым состояние в точках, где выполняется необходимое условие (9). Если при малом удалении от такой точки вектор $\mathrm{dN}$ всегда направлен обратно к ней, то состояние устойчиво, в противном случае - нет. Это хорошо видно на рисунках 1, a-1, г.

Дадим интерпретацию представленных на рис.1 четырёх вариантов развития сообществ. В варианте «а» первый вид является более сильным конкурентом в межвидовой борьбе и при любых начальных условиях полностью вытесняет второй вид, равновесная точка - $\mathrm{N}_{1}=\mathrm{K}_{1}, \mathrm{~N}_{2}=0$. В варианте «б» - обратная картина. В варианте «в» возможны две точки устойчивого равновесия: $\mathrm{N}_{1}=\mathrm{K}_{1}, \mathrm{~N}_{2}=0$ или $\mathrm{N}_{1}=0$, $\mathrm{N}_{2}=\mathrm{K}_{2}$. К какой из этих точек приведет процесс развития сообщества, зависит от начальных условий: вид, имеющий преимущество при $\mathrm{t}=0$, вытеснит конкурента полностью. Наконец в варианте «г» имеется единственное устойчивое состояние, в котором сосуществуют оба вида (точка пересечения прямых 1 и 2 на рис.1).

\section{Случай с одним лимитирующим ресурсом}

Применим описанный подход к анализу развития предприятия, на котором параллельно производятся два существенно различных вида продукции, которые конкурируют между собой. Для этого классические уравнения (8) запишем в предложенной выше форме уравнения (6), причём сначала будем считать, что производство обоих видов продукции лимитируется одним и тем же ресурсом S:

$$
\left\{\begin{array}{l}
\frac{d N_{1}}{d t}=\frac{r_{1} N_{1}\left(S-s_{1} N_{1}-s_{2} N_{2}\right)}{S} \\
\frac{d N_{2}}{d t}=\frac{r_{2} N_{2}\left(S-s_{1} N_{1}-s_{2} N_{2}\right)}{S}
\end{array}\right.
$$

Индексы 1и 2 относятся к 1-му и 2-му виду продукции соответственно.

Если перейти к обозначениям, принятым в системе (8), получим

$$
K_{1}=\frac{S}{s_{1}} ; K_{2}=\frac{S}{s_{2}} ; \alpha_{12}=\frac{s_{2}}{s_{1}} ; \alpha_{21}=\frac{s_{1}}{s_{2}} \text {. }
$$

Таким образом, несколько абстрактные коэффициенты межвидовой борьбы $\alpha_{12}$ и $\alpha_{21}$ приобретают ясный экономический смысл - они представляют собой отношение удельных потребностей обоих видов продукции в лимитирующем ресурсе, причём $\alpha_{12}$ $=1 / \alpha_{21}$. При таком соотношении параметров характеристические прямые 1 и 2 (рис.1) сливаются в одну, что означает существование бесконечно большого числа 
удовлетворяющих условию (9) сочетаний $\mathrm{N}_{1}$ и $\mathrm{N}_{2}$, соответствующих отрезку прямой в плоскости $\mathrm{N}_{1} \mathrm{~N}_{2}$ между точками $\mathrm{N}_{1}=\mathrm{K}_{1}, \quad \mathrm{~N}_{2}=0$ и $\mathrm{N}_{1}=0, \mathrm{~N}_{2}=\mathrm{K}_{2}$. Другими словами, ни один вид не может полностью вытеснить другой, а их конечное соотношение определяется начальными условиями (при $\mathrm{t}=0$ ).

Система уравнений (10) имеет простое аналитическое решение

$$
\frac{N_{2}}{N_{20}}=\left(\frac{N_{1}}{N_{10}}\right)^{r_{2} / r_{1}}
$$

Некоторые результаты расчётов по этой формуле представлены на рисунке 2. Как видно, при $\mathrm{r}_{2} / \mathrm{r}_{1}>1$ первоначальное соотношение $\mathrm{N}_{20} / \mathrm{N}_{10}$ неуклонно изменяется в пользу второго вида продукции и чем больше отношение $\mathrm{r}_{2} / \mathrm{r}_{1}$, тем быстрее происходит это изменение. При $\mathrm{r}_{2} / \mathrm{r}_{1}<1$ процесс протекает, наоборот, в сторону уменьшения отношения $\mathrm{N}_{2} / \mathrm{N}_{1}$. Если же эффективность обоих производств одинакова $\left(\mathrm{r}_{2} / \mathrm{r}_{1}=1\right)$, то и соотношение между двумя видами продукции со временем не изменится (меняется только их абсолютный объём). Величина располагаемого ресурса $\mathrm{S}$, от которой зависит положение характеристической прямой $\mathrm{K}_{1} \mathrm{~K}_{2}$, на вид траекторий развития влияния не оказывает. Однако, чем больше $\mathrm{S}$ (и соответственно $\mathrm{K}_{1}$ и $\mathrm{K}_{2}$ ), тем больше возможностей для развития процесса и тем больше может проявиться преимущество одного вида продукции над другим.
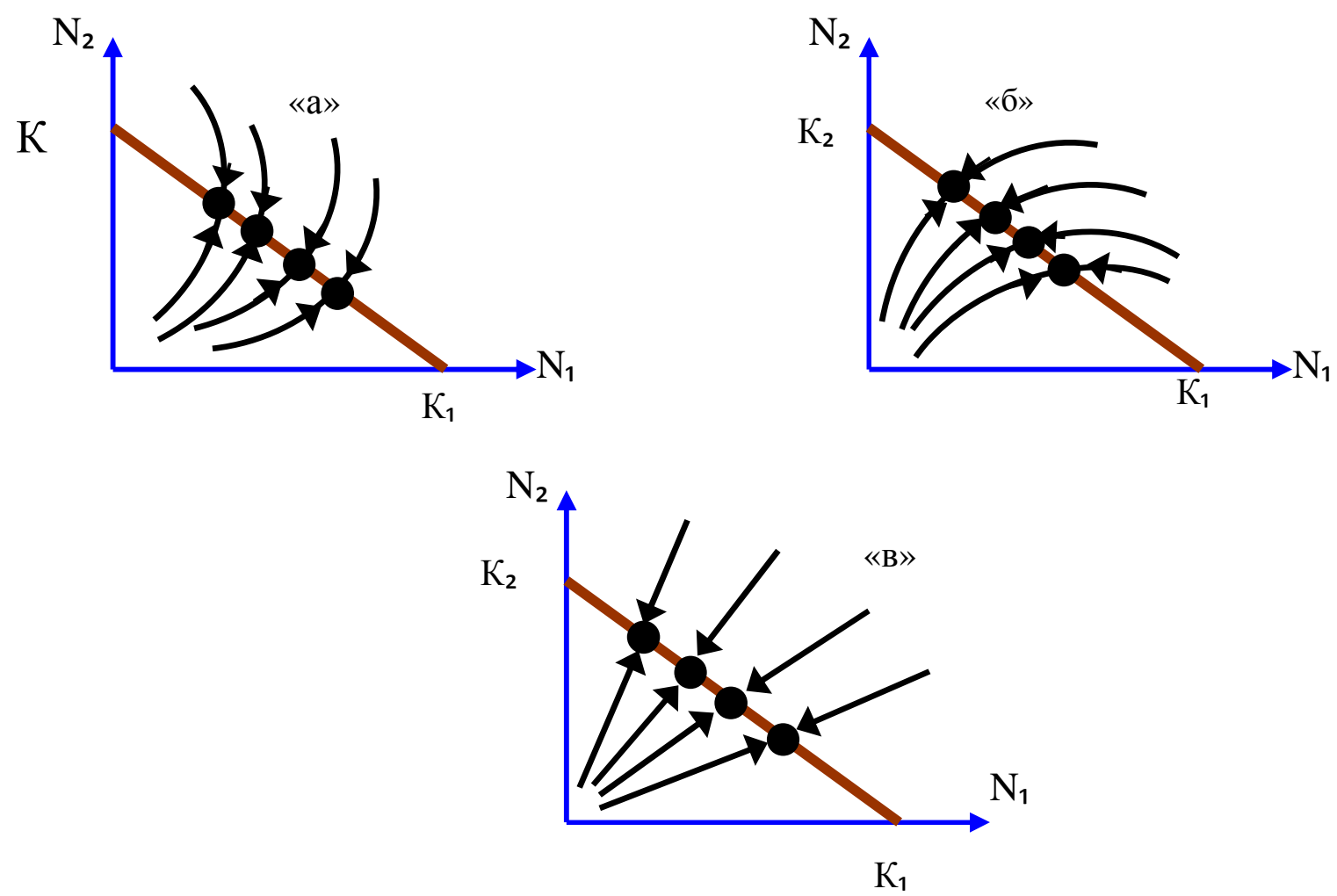

Рисунок 2 - Траектории развития при одном лимитирующем ресурсе для различных соотношений эффективности производства разных видов продукции: 


$$
\text { «a»- } \mathrm{r}_{2} / \mathrm{r}_{1}>1 ; \quad \quad \ll \sigma »-\mathrm{r}_{2} / \mathrm{r}_{1}<1 ; \quad \quad \ll \mathrm{B} »-\mathrm{r}_{2} / \mathrm{r}_{1}=1
$$

В полученном решении подразумевается, что оба вида продукции хотя бы в минимальном объёме уже производятся $\left(\mathrm{N}_{10}>0, \mathrm{~N}_{20}>0\right)$. В действительности же бывает, что специфика предприятия не позволяет производить другой вид продукции. Выходом может быть диверсификация, которая требует определённых затрат и времени для подготовки, и лишь после этого можно рассматривать конкурентное развитие двух видов производств и применять предложенную модель.

Оценим устойчивость состояний, соответствующих характеристической прямой $K_{1} K_{2}$ (на рисунке 2 обозначены точками). Эти состояния можно охарактеризовать термином «безразличное равновесие». Если в результате некоторого внешнего воздействия система переходит из одного состояния в другое вдоль прямой $\mathrm{K}_{1} \mathrm{~K}_{2}$, то не возникает никаких факторов, стремящихся вернуть её обратно (как при устойчивом равновесии) или удалить её ещё дальше (как при устойчивом равновесии). То есть система стабильно находится в новом положении, пока на неё не будет оказано какоелибо другое внешнее воздействие.

Пусть по-прежнему система находится в равновесии (точки на прямой $\mathrm{K}_{1} \mathrm{~K}_{2}$ ). Но внешнее воздействие таково, что точка состояния системы отходит от прямой $\mathrm{K}_{1} \mathrm{~K}_{2}$. Очевидно, затем произойдёт возврат на ту же прямую, но практически всегда в другую точку. Например, если увеличить $\mathrm{N}_{1}$ или уменьшить $\mathrm{N}_{2}$, то система вернётся на прямую $\mathrm{K}_{1} \mathrm{~K}_{2}$ в точку, расположенную ниже исходной (ближе к $\mathrm{K}_{1}$ ). Соотношение $\mathrm{N}_{1}$ и $\mathrm{N}_{2}$ изменится в пользу $\mathrm{N}_{1}$, причём этот качественный сдвиг сохранится и после возврата системы в равновесное состояние (на прямую $\mathrm{K}_{1} \mathrm{~K}_{2}$ ). При увеличении $\mathrm{N}_{2}$ или уменьшении $\mathrm{N}_{1}$ результат будет прямо противоположным.

Несколько слов о состояниях системы $\mathrm{N}_{1}, \mathrm{~N}_{2}$, находящихся справа от прямой $\mathrm{K}_{1} \mathrm{~K}_{2}$. Формально они не имеют смысла, так как для их реализации необходим ресурс больше максимально возможного $(\mathrm{S})$. Однако в действительности такую ситуацию можно представить. Например, используются материалы и комплектующие из нормативного запаса, обслуживающий персонал работает сверхурочно и т.п. Но зато после этого неизбежно наступает спад производства (нужно восстановить запас, дать отгулы рабочим), что и показывает направление стрелок на рисунке 2.

Полученные зависимости, с одной стороны позволяют предсказать ход развития производства конкурирующих видов продукции, а с другой стороны показывают возможности целенаправленного регулирования этого процесса. Будем считать, что начальные значения $\mathrm{N}_{10}$ и $\mathrm{N}_{20}$ - это объективная реальность, т.е. то, что мы имеем на сегодняшний день. Тогда развитие предприятия можно планировать по нескольким различным вариантам.

Наиболее типичный вариант (с точки зрения интересов государства) обеспечение требуемого объёма продукции госзаказа 


\section{Случай, когда лимитирующие ресурсы разные}

Рассмотрим развитие двух видов конкурирующих внутри одного предприятия производств в случае, когда они лимитируются разными видами ресурсов: $\mathrm{S}$ - ресурс, лимитирующий первый вид производства, например, производственные площади, L второй вид, например, производственный персонал. Обозначим удельные потребности обоих производств в ресурсе $S$ через $\mathrm{s}_{1}$ и $\mathrm{s}_{2}$, а в ресурсе $l_{1}$ и $l_{2}$ соответственно. Тогда основная система уравнений будет выглядеть так

$$
\left\{\begin{array}{l}
\frac{d N_{1}}{d t}=\frac{r_{1} N_{1}\left(S-s_{1} N_{1}-s_{2} N_{2}\right)}{S} \\
\frac{d N_{2}}{d t}=\frac{r_{2} N_{2}\left(L-l_{1} N_{1}-l_{2} N_{2}\right)}{L}
\end{array}\right.
$$

В обозначениях, принятых в системе (8), получим

$$
K_{1}=\frac{S}{s_{1}} ; K_{2}=\frac{L}{l_{2}} \quad \alpha_{12}=\frac{s_{2}}{s_{1}} ; \alpha_{21}=\frac{l_{1}}{l_{2}} \text {. }
$$

Здесь также, как и в случае с одним лимитирующим ресурсом, коэффициенты $\alpha_{12}$ и $\alpha_{21}$ имеют ясный экономический смысл, однако прямая связь между ними отсутствует. Кроме этого, с учетом условия (7) имеем

$$
\frac{S}{s_{1}} \prec \frac{L}{l_{1}}, \frac{L}{l_{2}} \prec \frac{S}{s_{2}}
$$

Используя эти неравенства, легко показать, что для рассматриваемого случая развитие будет всегда происходить по варианту «г» (см. рисунок 1), для которого характерно наличие единственного устойчивого состояния, соответствующего точке пересечения характеристических прямых $\mathrm{dN}_{1} / \mathrm{dt}=0$ и $\mathrm{dN}_{2} / \mathrm{dt}=0$. Значения N1k и N2к, соответствующие этой точке, вычисляются через четыре параметра (13)

$$
N_{1 k}=\frac{\frac{S}{s_{2}}-\frac{L}{l_{2}}}{\frac{s_{1}}{s_{2}}-\frac{l_{1}}{l_{2}}} ; \quad N_{2 k}=\frac{\frac{L}{l_{1}}-\frac{S}{s_{1}}}{\frac{l_{2}}{l_{1}}-\frac{s_{2}}{s_{1}}}
$$

Учитывая неравенства (14), а также вытекающие из них неравенства

$1_{1} / s_{1}<\mathrm{L} / \mathrm{S}, \mathrm{l}_{2} / \mathrm{s}_{2}>\mathrm{L} / \mathrm{S}, \mathrm{l}_{2} / \mathrm{s}_{2}>\mathrm{l}_{1} / \mathrm{s}_{1}, \mathrm{l}_{2} / \mathrm{l}_{1}>\mathrm{s}_{2} / \mathrm{s}_{1}, \mathrm{~s}_{1} / \mathrm{s}_{2}>\mathrm{l}_{1} / \mathrm{l}_{2}$

можно утверждать, что искомая точка пересечения действительно существует и лежит в первом квадранте плоскости $\mathrm{N}_{1} \mathrm{~N}_{2}$.

Таким образом для рассматриваемого случая установлен весьма любопытный факт - независимо от начальных условий $\mathrm{N}_{10}, \mathrm{~N}_{20}$ и от коэффициентов эффективности $\mathrm{r}_{1}, \mathrm{r}_{2}$ система придёт в единственно возможное устойчивое состояние $\mathrm{N} 1 \mathrm{k}, \mathrm{N} 2 \kappa$, определяемое соотношениями (15). Координаты начальной точки и параметры $\mathrm{r}_{1}, \mathrm{r}_{2}$ влияют на форму траектории развития, на время достижения конечной точки, но не на координаты последней. Типичные траектории развития представлены на рисунке 3. 
Для целенаправленного управления развитием предприятия в данном случае имеется только одна возможность изменять суммарные объёмы лимитирующих ресурсов $\mathrm{S}$ и L. Необходимые изменения в зависимости от нужного конечного результата (N1k, N2к) определяются с помощью формул (15). Механизмом реализации такого способа управления, как уже упоминалось ранее, может служить выделение целевых кредитов.

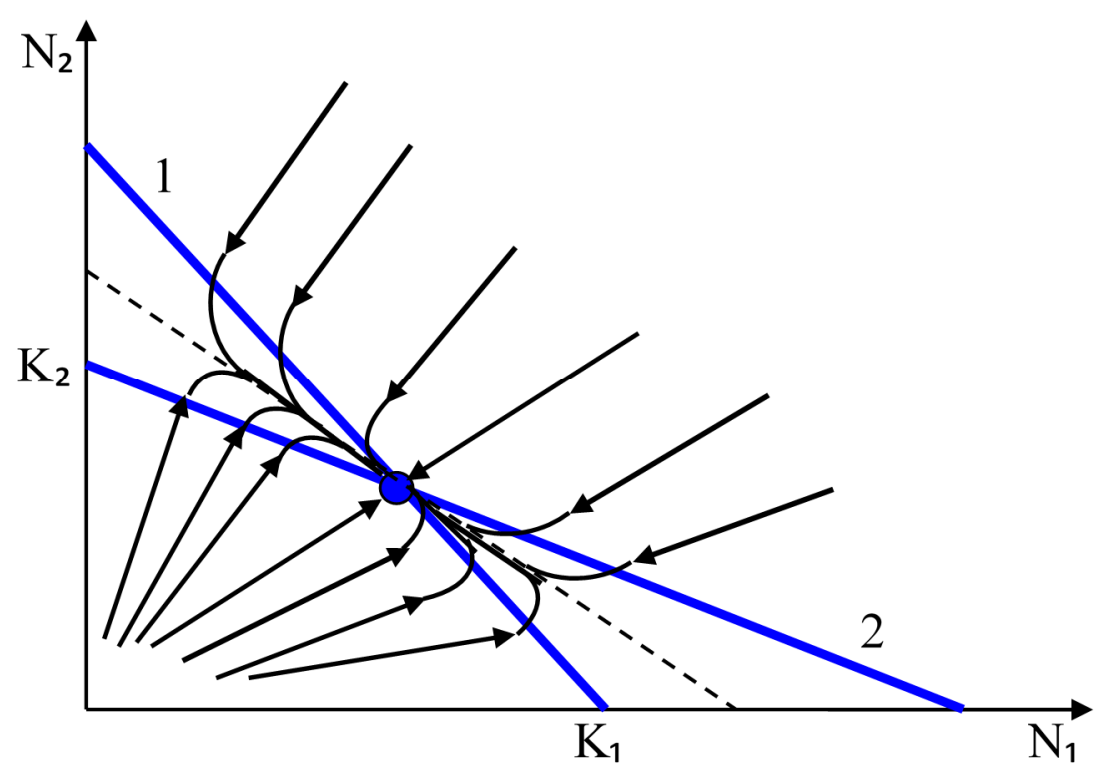

Рисунок 3 - Траектории развития при двух различных лимитирующих ресурсах

$$
1-\mathrm{dN}_{1} / \mathrm{dt}=0 \quad 2-\mathrm{dN}_{2} / \mathrm{dt}=0
$$

\section{Учёт дополнительных факторов}

Сравнение рассмотренных вариантов развития предприятия с вариантами развития биологических сообществ (см. рисунок 1) показывает, что в первом случае картина значительно более однообразная - или достижение безразличного равновесия, или единственного устойчивого состояния. Чем это обусловлено? Дело в том, что мы до сих пор сводили конкуренцию между видами продукции только к борьбе за ресурсы. Поэтому относительные вариации определяющих параметров $K_{1}, K_{2}, \alpha_{12}$ и $\alpha_{21}$ оказались ограниченными и довольно тесно связанными между собой. В биологических же системах (в уравнениях 8) эти параметры могут принимать практически любые значения. В них понятие конкурентной борьбы значительно шире, чем просто борьба за ресурсы.

Но и в производственных системах в реальной жизни действует множество дополнительных факторов создающих те или иные предпочтения.

Для качественной оценки влияния дополнительных факторов введём некоторый обобщённый параметр $\psi>1$, отражающий преимущества одного вида продукции над другим. Тогда, например для случая с одним лимитирующим ресурсом систему уравнений (10) можно заменить на следующую: 


$$
\left\{\begin{array}{l}
\frac{d N_{1}}{d t}=\frac{r_{1} N_{1}\left(S-s_{1} N_{1}-\psi \cdot s_{2} N_{2}\right)}{S} \\
\frac{d N_{2}}{d t}=\frac{r_{2} N_{2}\left(S-(1 / \psi) s_{1} N_{1}-s_{2} N_{2}\right)}{S}
\end{array}\right.
$$

Здесь считается, что преимущество имеет продукция второго вида. Поэтому коэффициент межвидовой конкуренции $\alpha_{12}$ (подавление 1-го вида вторым) увеличивается $\alpha_{12}=\psi \mathrm{s}_{2} / \mathrm{s}_{1}$, а коэффициент $\alpha_{21}$ (подавление 2-го вида первым) уменьшается $\alpha_{21}=(1 / \psi) \mathrm{s}_{1} / \mathrm{s}_{2}$.

В результате введения коэффициента $\psi>1$ решение системы уравнений (17) существенно отличается от полученного выше для системы уравнений (10). Так как $\mathrm{K}_{1}$ $=\mathrm{S} / \mathrm{s}_{1}<\mathrm{K}_{2} / \alpha_{21}=\left(\mathrm{S} / \mathrm{s}_{2}\right)\left(\mathrm{s}_{2} / \mathrm{s}_{1}\right) \psi, \mathrm{K}_{2}=\mathrm{S} / \mathrm{s}_{2}>\mathrm{K}_{1} / \alpha_{12}=\left(\mathrm{S} / \mathrm{s}_{1}\right)\left(\mathrm{s}_{1} / \mathrm{s}_{2}\right)(1 / \psi)$, то система будет развиваться по варианту «б» (см. рис.1). В результате независимо от начальных условий второй вид вытеснит первый. Причём это произойдёт даже при сколь угодно малом превышении $\psi$ над единицей. Такое сильное влияние $\psi$ на развитие процесса объясняется тем, что при $\psi=1$ система хотя и находится в равновесии, но оно, как отмечалось выше, - «безразличное». Поэтому даже незначительное, но стабильное дополнительное преимущество 2-го вида продукции ( $\psi>1)$ обеспечивает беспрепятственное перемещение точки равновесия (N1k, N2к) вдоль характеристической прямой в направлении точки $\mathrm{N}_{1}=0, \mathrm{~N}_{2}=\mathrm{K}_{2}$.

Как с этим бороться, особенно в случае, когда речь идёт о социально значимой продукции? Производство этой продукции должно стать таким же привлекательным, как и продукции вида 2 (чтобы было $\psi=1$ ). Для этого необходимо вводить специальные меры стимулирования. Хотя сами коэффициенты $\mathrm{r}_{1}$ и $\mathrm{r}_{2}$ в правой части уравнений (17) на характер решения (расположение характеристических прямых) не влияют, но опосредованно увеличение $\mathrm{r}_{1}$ должно уменьшить коэффициент $\psi$. Причина в том, что повышение рентабельности производства социально значимой продукции сделает её более привлекательной для предприятия.

Теперь введём коэффициент $\psi$ в систему уравнений (12):

$$
\left\{\begin{array}{l}
\frac{d N_{1}}{d t}=\frac{r_{1} N_{1}\left(S-s_{1} N_{1}-\psi \cdot s_{2} N_{2}\right)}{S} \\
\frac{d N_{2}}{d t}=\frac{r_{2} N_{2}\left(L-(1 / \psi) l_{1} N_{1}-l_{2} N_{2}\right)}{L}
\end{array}\right.
$$

В результате получим $\alpha_{12}=\psi \mathrm{s}_{2} / \mathrm{s}_{1}, \alpha_{21}=(1 / \psi) 1_{1} / l_{2}$. С учётом этого, а также неравенств (14) имеем

$\mathrm{K}_{1}=\mathrm{S} / \mathrm{s}_{1}<\mathrm{K}_{2} / \alpha_{21}=\left(\mathrm{L} / \mathrm{l}_{2}\right)\left(\mathrm{l}_{2} / \mathrm{l}_{1}\right) \psi ; \mathrm{K}_{2}=\mathrm{L} / \mathrm{l}_{2} \lessgtr \mathrm{K}_{1} / \alpha_{12}=\left(\mathrm{S} / \mathrm{s}_{1}\right)\left(\mathrm{s}_{1} / \mathrm{s}_{2}\right)(1 / \psi)$.

Знак $\lessgtr$ во втором неравенстве показывает, что при не очень больших значениях $\psi$ (например $\psi=1$ ) реализуется знак «меньше». Тогда, как и для системы (12), развитие 
будет происходить по варианту «г», система будет стремиться к единственно устойчивому состоянию. Если же коэффициент $\psi$ достаточно велик, т.е. $\psi>\left(\mathrm{S} / \mathrm{s}_{2}\right)\left(\mathrm{l}_{2} / \mathrm{L}\right)$ $>1$ (последнее следует из 2-го неравенства (14)), то развитие системы пойдёт по варианту «б», как для системы (17)) с одним лимитирующим ресурсом.

Сравнение решений уравнений (17) и (18), в которые введён параметр $\psi$, показывает, что при наличии двух различных лимитирующих ресурсов процесс развития системы более устойчив в смысле сохранения продукции 1-го вида. Она не будет вытеснена вторым видом даже при условии $1<\psi<\left(\mathrm{S} / \mathrm{s}_{2}\right)\left(\mathrm{l}_{2} / \mathrm{L}\right)$, в то время как в системе с одним лимитирующим ресурсом выпуск продукции 1-го вида сводится к нулю при $\psi>1$.

\section{Возможные пути развития модели}

Предложенный подход может быть применён для изучения довольно широкого круга экономических проблем, помимо рассмотренных выше. В частности, это относится к конкурентной борьбе за рынки сбыта, к совместному развитию производства нескольких видов продукции, к обновлению номенклатуры производимой продукции и т.д. Наличие рынка сбыта является одним из естественных ограничений развития производства, которое в математическом смысле полностью аналогично рассмотренным выше ограничениям по ресурсам. Задаётся оно величиной спроса К, при этом, очевидно, должно быть $\mathrm{N} \leq \mathrm{K}$. И если лимитирующим для развития производства является рынок сбыта, то в уравнениях развития типа (6) вместо отношения S/s должен фигурировать спрос К.

Специально данное ограничение нами не рассматривалось, но фактически оно входит во все основные уравнения - (5), (10) и (12) наряду с ограничениями по ресурсам. Поэтому, например уравнения (10) можно использовать для анализа развития нескольких предприятий, производящих одинаковую продукцию и сбывающих её на общем рынке (если предприятий больше двух, то соответственно увеличивается количество уравнений). Эти же уравнения описывают и развитие одного предприятия, на котором производятся две модификации одного изделия - новая и старая, сбываемые на одном рынке.

Учёт изменения спроса на продукцию во времени является одним из важнейших принципов в разработке стратегии развития предприятия. Если на основании прогноза определён вид функции $\mathrm{K}(\mathrm{t})$, то тогда для решения этой задачи также можно использовать уравнения (10), хотя решение этих уравнений несколько усложняется по сравнению со случаем, когда $\mathrm{K}=$ const.

В некоторых случаях приведенные выше уравнения могут потребовать уточнения. Дело в том, что в п.1 было сделано допущение об отсутствии временной задержки между вложением средств и соответствующим расширением производства. Однако, если проводится серьёзная перестройка производства, такое допущение может привести к большим погрешностям. Более правильно будет использовать уравнения с запаздыванием во времени [2]. Например вместо уравнения (5) следует использовать уравнение 


$$
\frac{d N(t)}{d t}=\frac{r N(t-\tau)(K-N(t-\tau))}{K}
$$

где $\tau$ - запаздывание во времени.

Смысл этого уравнения состоит в следующем. Средства, вложенные в расширение производства, начинают давать отдачу только через период времени $\tau$. При этом внешние ограничения (наличие производственных площадей и т.п.), влияющие на эффективность вложений (дробь в правой части уравнения), относятся к тому же моменту времени, когда средства вкладывались. Если же эти ограничения сказываются только с момента реализации вложенных средств (ограничения по сырью, производственному персоналу, рынку сбыта), то форма уравнения будет несколько иная

$$
\frac{d N(t)}{d t}=\frac{r N(t-\tau)(K-N(t))}{K}
$$

Использование более строгих уравнений (19) и (20) совместно с прогнозируемым изменением спроса на продукцию $\mathrm{K}(\mathrm{t})$ позволяет подойти к решению ещё более сложной проблемы - диверсификации производства, выбору сочетаний видов продукции, наивыгоднейших с учётом изменения рыночной конъюнктуры со временем. конечно, в данном случае предприятие уже не может рассматриваться как простой «биологический» объект. В математической модели необходимо будет учитывать элементы планирования, целенаправленное перераспределение прибыли между разными производствами. Однако основные уравнения останутся без изменений.

Приведенные примеры показывают, что предложенная математическая модель развития предприятий, основанная на биологических аналогиях, может иметь значительно более широкую область применения, чем это следует из первоначальной постановки задачи. Хотя при этом навряд ли удастся получить столь простые и наглядные результаты, как в данной работе, но всё же математические решения - в виде численного анализа вариантов или имитационного моделирования - вполне смогут использоваться на практике.

В заключение отметим, что в качестве объекта моделирования в предлагаемом методе не обязательно рассматривать именно предприятие. Это может быть и группа предприятий, и регион, и отрасль, и даже группа отраслей. Главное - чтобы такой комплексный объект характеризовался некоторой общей целью развития и для её достижения мог бы управляться как единое целое. Параметры, фигурирующие в уравнениях развития, определяются при этом путем осреднения по элементам объекта.

\section{Список информационных источников}

[1] Бигон М., Харпер Дж., Таунсенд К. Экология. Особи, популяции и сообщества, т.1. М.: Мир, 1989. 667 с.

[2] Свирижев Ю.М., Логофет Д.О. Устойчивость биологических сообществ. М.: Наука, 1978. 375 с. 
[3] Остроух А.В., Синха Бабу Раджа Исследование информационных систем управления взаимоотношениями с поставщиками // Автоматизация и управление в технических системах. - 2013. - № 4.1; DOI: 10.12731/2306-1561-2013-4-10; URL: auts.esrae.ru/6-124 (дата обращения: 22.11.2013).

[4] Остроух А.В. Информационные технологии в научной и производственной деятельности / [ред. А.В. Остроух] - М: ООО "Техполиграфцентр", 2011. - 240 с. ISBN 978-5-94385-056-1.

[5] Остроух А.В. Исследование начального периода моделирования на точность среднеинтегральной оценки имитационных моделей / А.В. Остроух, А.А. Солнцев, Н.В. Солдатов, К.А. Новицкий, П.С. Якунин // Вестник МАДИ - 2010. Вып. 2(21). - С. 61-65.

[6] Николаев А.Б. Информационные технологии в менеджменте и транспортной логистике: учебное пособие / А.Б. Николаев, А.В. Остроух. - Saint-Louis, MO, USA: Publishing House Science and Innovation Center, 2013. - 254 c. - ISBN 978-0615-67110-9. 